\title{
Design Approach for Touch Based User Interfaces
}

\author{
Hyun-Chul Lee \\ Korea Atomic Energy Research Institute \\ PO BOX 105, Yuseong, Daejeon, \\ South Korea \\ +82-42-868-2942 \\ leehc@kaeri.re.kr
}

\author{
Jung-Woon Lee \\ Korea Atomic Energy Research Institute \\ PO BOX 105, Yuseong, Daejeon, \\ South Korea \\ +82-42-868-8322 \\ leejw@kaeri.re.kr
}

\begin{abstract}
Touch based interfaces are commonplace these days. A design approach for touch based user interfaces carried out in the course of developing a digital system for nuclear power plants is introduced in this paper. A style guide and human factors experiment play an important role in designing touch based user interfaces.
\end{abstract}

\section{Categories and Subject Descriptors}

H.5.2 [Information Interfaces and Presentation]: User Interfaces - Ergonomics, Evaluation/methodology, Graphical User Interfaces, Input devices and strategies, Interaction styles, Screen design, Style Guides, User-centered design.

\section{General Terms}

Management, Measurement, Performance, Design, Reliability, Experimentation, Human Factors, Standardization

\section{Keywords}

Touch screen, Style Guide, Human Factors

\section{INTRODUCTION}

Nowadays it is very easy to find a touch-based user interface in real life. Automated teller machine (ATM) in a public place is a computerized device for financial transactions and provides a typical touch-based user interface. Required information is retrieved through touch screens in the information booths of museums or government and public offices.

Touch based user interfaces are becoming popular in the nuclear industry as digitalized systems replace analog based systems. Though various touch based user interfaces are available in the nuclear industry, it seems that human factors are taken into account less in the interface design stage because touch based user interfaces have been applied to the non-safety systems and infrequently-used systems rather than safety systems and frequently-used systems.

A design approach for touch based user interfaces carried out in the course of developing a digital system for nuclear power plants is introduced in this paper.

\section{THE DIGITAL SYSTEM}

A Korean project group, KNICS, developed a new digitalized reactor protection system (RPS) and the developed system is composed of three cabinets with several racks. One of the three cabinets of an RPS is used for the RPS function testing and monitoring by maintenance operators and is equipped with a flat panel display (FPD) with a touch screen capability as a main user interface for operations. In general, maintainers use the FPD to test the RPS function periodically, at one month intervals. The FPD is a color LCD.

The characteristics of the hardware and software development environments for the RPS FPD limit the design of touch based user interfaces.

Main design constraints are as follows:

- Hardware: Physical size and resolution of the FPD

- Software: OS and graphic environment/library

\section{DESIGN APPROACH}

\subsection{Design Guidelines and a Style Guide}

There are many human factors design guidelines and standards published by governments, organizations, companies, and even individuals. As a result, user interface designers will be confronted with a tremendous quantity of guideline items related to a Visual Display Unit (VDU). However, design guideline items suitable for an FPD or touch screen design are not many.

Button size and spacing are mainly addressed in the design guidelines. However design guides related to the specific design variables such as a coloring, button dynamics, pop-up window, information allotment, screen splitting, and so on, are not sufficiently provided.

Thus a style guide for the FPD was developed [1]. The overall development procedure of the RPS FPD user interface style guide was composed of an information acquisition, selection and screening, property determination and discussion, as well as a conversion and review.

The first development step towards the style guide is to gather information about the RPS and the RPS FPD. What are the RPS functions and what role the RPS FPD plays are a basic concern in order to proceed with the development of a style guide because these functions and the user interfaces can not be considered separately. As a result, we refined the items to be explicitly determined in the style guide such as what screen layout could be expected, which design elements could be used in the screens, 
what color scheme could be applied, what manners of navigation were required, etc.

Various human factors guidelines related to the VDU were gathered and a database management system (DBMS) of those guidelines was developed. Through the DBMS, guideline and guideline items selections were performed. Every design variable and design element defined in a prior step was entered and the search results were repeatedly merged.

The human factors design guidelines rarely provide a specific or optimal value for a design variable (e.g. button) or design element (e.g. line spacing) [2]. They often give the recommended range, minimum value, or maximum value. However designers want to obtain not an acceptable range but a specific value in a style guide. With respect to each design element we suggested the specific values and discussed them with the system designers and user interface designers in order to verify that the value could be applicable to all the screens.

If a designer wants to draw a pushbutton group on a touch screen, he has to decide the dimension applicable to all the pushbuttons in the group. In the case that the designer sets the dimension to 30 $\mathrm{mm}$, it is not enough in most situations because the designer uses a design software tool which accepts its own metric (e.g. point) not a millimeter scale. So does the RPS FPD design software tool. Therefore we transformed the value in terms of the software tool so that designers could use the value without any conversion.

\subsection{Experiments and User Evaluation}

To ensure that the RPS FPD drawn using the style guide supports the maintenance personnel tasks, a human factors experiment with task scenarios and a design review by human factors experts were implemented [3]. The task scenarios were prepared to cover every representative task defined in the task analysis. Subjects having experience in maintaining the RPS of a nuclear power plant participated in the human factors evaluation. They were trained in maintenance tasks on the KNICS RPS cabinet and FPD before the scenario imposition. The order of the experimental runs was completely randomized to eliminate a bias caused by an ordering. During the experiment, every subject behavior was recorded using a video camera. Subject's performance was scored a success or a failure according to a task's completeness. As for the results of the performance analysis, it was verified that most of the task scenarios were completed fully by the subjects. As for the results of the experiments, many design concerns with the RPS FPD were deduced and classified into a mandatory or a recommendation according to the importance of the related tasks.

After the experiments and design improvements, user evaluation by real plant maintenance personal was carried out. Subjects were required to perform their tasks during the periodic maintenance work and to evaluate the efficiency of the FPD.

\subsection{Integrated Style Guide}

Standardization has many profits: cost, time, manpower, etc. There are three protection systems in nuclear power plants: the RPS, the ESF-CCS (Engineered Safety Features-Component Control System), and the CPC (Core Protection Calculator). The ESF-CCS and the CPC are also equipped with an FPD with a touch capability. Thus the style guide for the RPS FPD can be applied to the design of the ESF-CCS and the CPC.
It is possible that one FPD could provide a combined touch based user interface for the RPS, the ESF-CCS, and the CPC. This case requires an integrated style guide in order to provide unified look and feel effects throughout the three systems.

Many design variables could remain without changes. A new unified menu system and coloring method were proposed for the integrated style guide (refer to Figure 1.).

\section{CONCLUSIONS}

In the design of the touch based user interfaces, it is desirable:

(1) to develop a style guide

- Design guidelines provide insufficient information.

- Hardware and software constraints should be considered.

- Application to another system is easy

- It makes an integration with other designs easy.

- Design verification is simple

(2) to undertake a human factors experiment

- Real users can confirm the design and the style guide

- Design issues can be resolved on the basis of professional system knowledge

- Recommendations for a design improvement can be suggested

\section{REFERENCES}

[1] Hyun-Chul Lee, et. al., A User Interface Style Guide for the Reactor Protection System Cabinet Operator Module, In Proceedings of 2004 NPIC\&HMIT, Sep, 2004, Ohio State Univ., $\mathrm{OH}$.

[2] O'Hara, J.M., et al., Human-System Interface Design Review Guidelines, NUREG-0700 Rev.2, May 2002

[3] Hyun-Chul Lee, et. al., Human Factors activities for Reactor Protection System Cabinet Development, In Proceedings of HAMMAHA 2005, July, 2005, San Diego, CA.

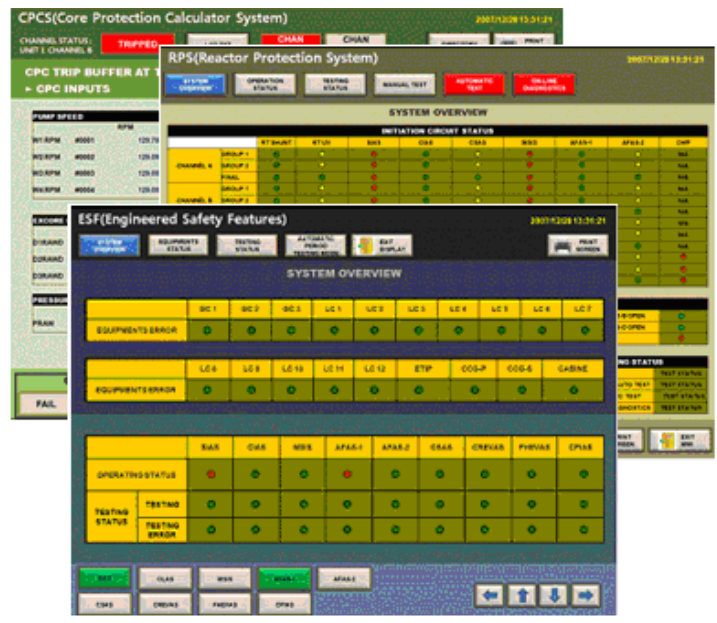

Figure 1. Screen captures of the three systems. 